\title{
Development of a mobile multimodal biosignal instrument for simultaneous measurement and analysis of four clinically relevant biosignals, obtained from both normal and pathological subjects
}

\begin{abstract}
This paper presents the development and construction of a multimodal biosignal instrument to monitor and analyse simultaneously for clinically relevant biosignals, obtained from both normal and pathological subjects. Biosignals were tapped from the appropriate locations of subjects' bodies and buffered by operational amplifiers through disposable electrodes. This was directly followed by pre-processing the generated signals, feeding their outputs into an analogue to digital converter (ADC) input of 40PIC18F4450 self programmed microcontroller, and then fed into ADS1299 amplifier with supporting logics $74 \mathrm{HC} 14$ and HEF4050 respectively. The signals obtained were transmitted through $869.95 \mathrm{MHz}$ frequency modulation transmitter with an improvised whip antenna, to stationary end consisting of FM receiver circuit connected to a 64 bit 4G RAM Computer, through a USB COM Port.
\end{abstract}

Keywords: biosignal, transmitter, receiver
Volume 4 Issue 3 - 2018

\author{
Adegbenro Sunday Ajani,' Olusope Michael \\ Alade, ${ }^{2}$ Oluwatayo Sandra Ajani, ${ }^{3}$ Oladosu \\ Jamiu Alabi ${ }^{4}$ \\ 'Department of Physics and Material Science, Kwara State \\ University, Nigeria \\ ${ }^{2}$ Department of Pure and Applied Physics, Ladoke Akintola \\ University of Technology, Nigeria \\ ${ }^{3}$ Ladoke Akintola University of Technology Teaching Hospital, \\ Nigeri \\ ${ }^{4}$ Osun State College of Technology, Nigeria
}

Correspondence: Ajani Adegbenro Sunday, Department of Physics and Material Sciences, Kwara State University, Malete, llorin, Nigeria, Email adegbenro.ajani@kwasu.edu.ng

Received: April 27, 20I8| Published: June 22, 2018

\section{Introduction}

Biomedical electronic applications, derived from biosignals, such as cortical mapping, seizure detection, conduction defects, diagnosis of ischemia, arrhythmia, neuromuscular problems, prosthesis and sleep studies, have received attention recently in clinical health practices. However, biosignals are of low frequencies and small amplitudes, with interference from environmental sources as a major challenge. Instrumentations for measurement and monitoring of these biosignals are in very limited supply, if not mostly unavailable, even in hospitals designed for modern health care in Nigeria. The existing ones in most clinical laboratories can only measure parameters for one type of biosignals; and they are quite expensive, due to the cost of construction and maintenance of the laboratories as well as payment of laboratory personnel. Another limitation is non-availability of wireless transmission of biosignal data from the laboratories directly to the medical experts expected to make medical decisions and diagnoses based on the outcome of the tests carried out. World Health Organization confirmed that each year a figure which represents 30 percent of the global cause of death, approximately estimated as 18 million people around the world died of cardiovascular and other related diseases in year 2017. ${ }^{1}$ Therefore, an instrument that accommodates multimodal signal evaluation and operates with sensor network wirelessly needs to be designed and made readily available to hospitals. Patients' health can then be monitored outside clinical environment such as offices and homes, if individuals or organizations have access to biosignal device that allows self monitoring. The wireless transmission will be expedient in the biosignal facility to enhance patient's mobility and transfer of real time clinical data, thus limiting patients' wheeling to - and - from the laboratories and related testing locations.

\section{Methodology}

The methods, steps and procedures employed in carrying out the construction and development of the multimodal biosignal system is discussed in this section.

\section{The development of the multimodal biosignal system}

The whole device has two main units, which are the mobile transmitting end and the stationary unit for reception. The transmitting unit is portable and it consists of all the channels to which the desired biomedical signals are multiplexed; it amplifies and transmits signals at an FM frequency of $869.85 \mathrm{MHz}$ through a locally made whip antenna. Ports are made on it, from which the improvised lead wires and disposable electrodes are connected. The receiving end on the other hand is a stationary unit, connected to the computer, with a programmable RX3A receiver module. The signals received are filtered and stored on the computer, ready for acquisition and analysis. Figure $1 \&$ Figure 2 describes the block diagram of the stages employed for the two units of the system. The four biosignal quantities measured were the Electromyogram, Electrocardiogram, Electroencephalogram and Speech signals. The transduction was achieved using the matching disposable electrode clipped on the tip of the lead wires, to be affixed to the recommended locations on the subjects where the signals are expected to be tapped. The signal preprocessed unit had the following components and specifications:

i) A wide band single supply operational amplifier that boosts and filters the output signal of the transducer.

I. TX3A 869.85 MHz FM transmitter as the front unit of the radio link. 
II. A 40PIC18F4450 microcontroller with USART and analogue to VIII. Disposable electrodes. digital converter.

III. An ADS1299 front end amplifier.

IV. Signal conditioning circuit.

V. Supporting logics $74 \mathrm{HC} 14$ and HEF4050

VI. A 32 Gigabytes micro SD storage card

VII. Lead wires
IX. The receiving end of the system on the other hand, had two components and specifications:

X. RX3B frequency modulation receiver

XI. MAX 232 with a standard interface and dual driver

XII. Digital module interface with USB COM port.

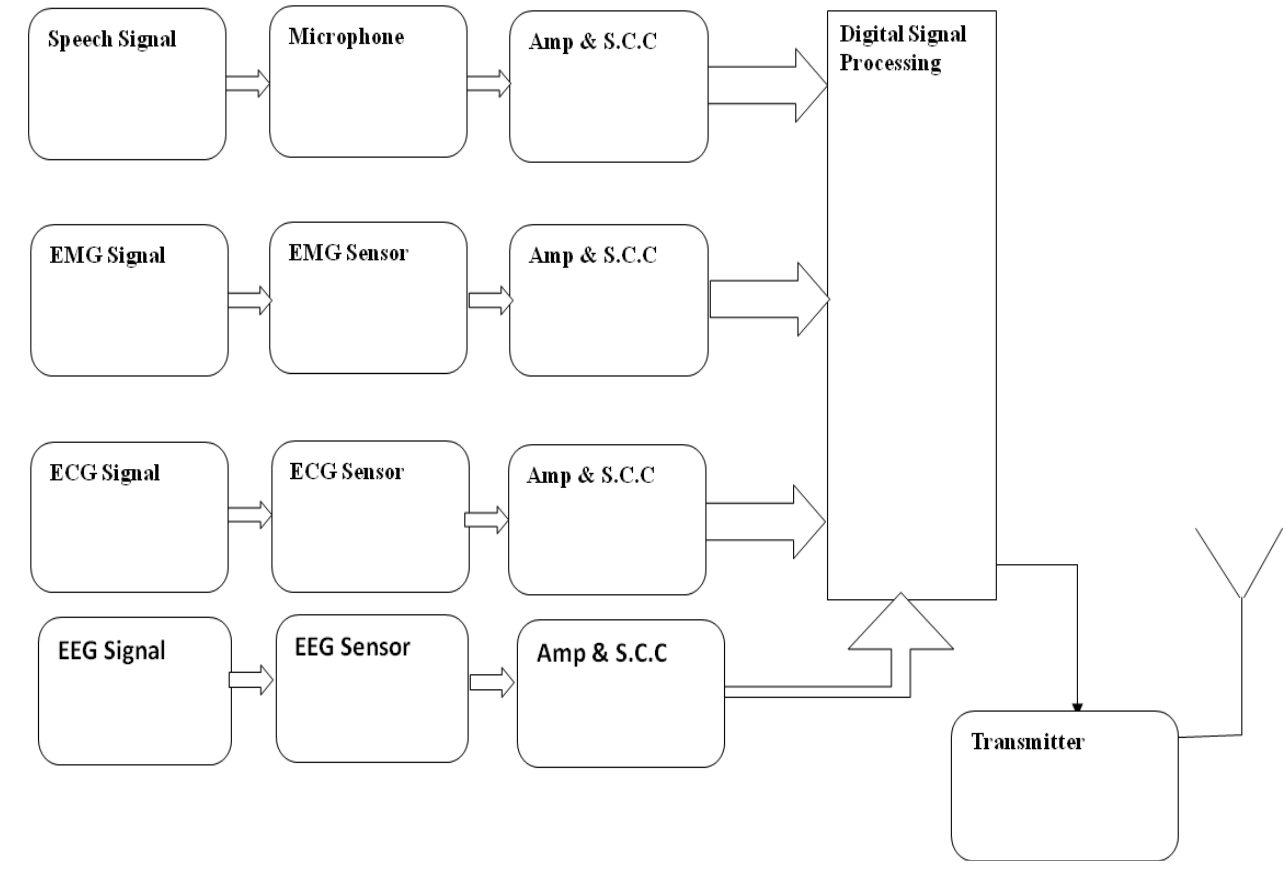

Figure I Block Diagram of Basic Transmitting Components.

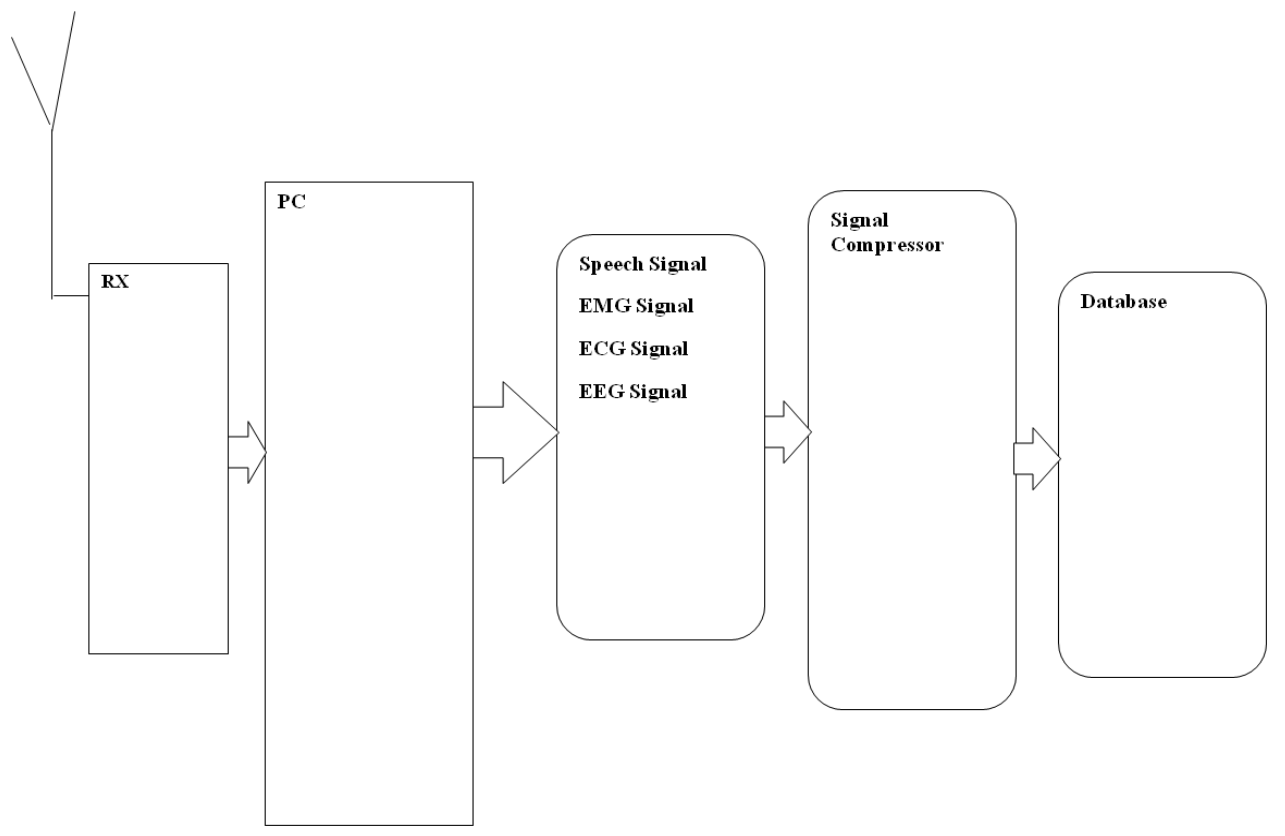

Figure 2 Block Diagram of Receiving End.

Citation: Ajani AS,Alade OM,Ajani OS, et al. Development of a mobile multimodal biosignal instrument for simultaneous measurement and analysis of four clinically relevant biosignals, obtained from both normal and pathological subjects. Int J Biosen Bioelectron. 20I8;4(3): I48-I57. DOI: I0.15406/ijbsbe.2018.04.00II7 


\section{The wireless transmitting end}

The transmitting unit is mobile and it consists of the following:

a) The frequency modulation transmitter

b) Microcontroller with the signal processing unit.

c) ADS1299 front end amplifier with other supporting logics

d) An Helical antenna.

e) SD card, lead wire and disposable electrodes

f) LCD

The pictorial view of the mobile transmitting section after construction is shown in Figure 3 \& Figure 4.

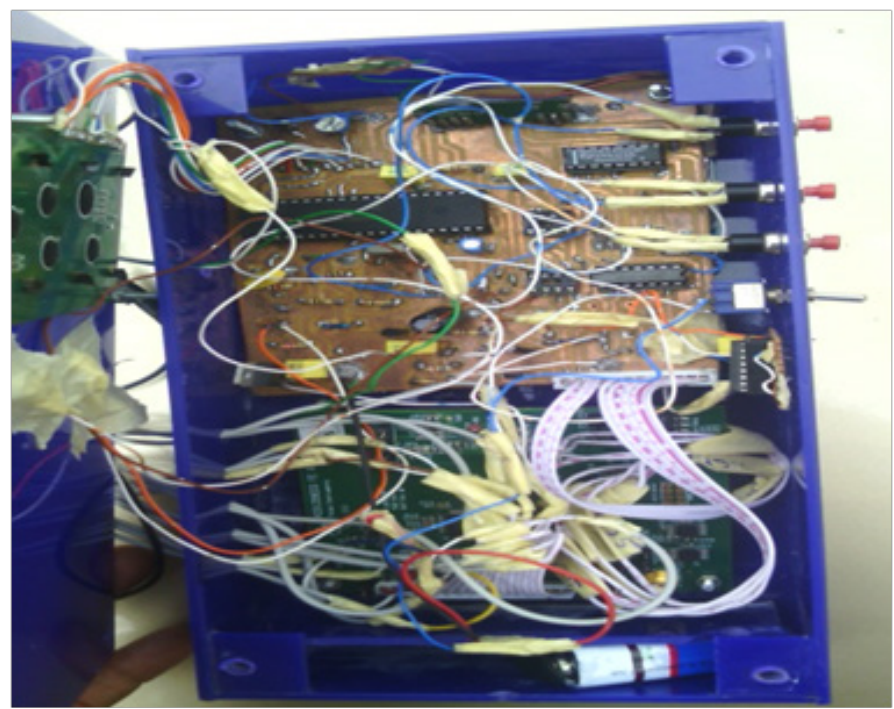

Figure 3 Internal Circuits of the Mobile Transmitter

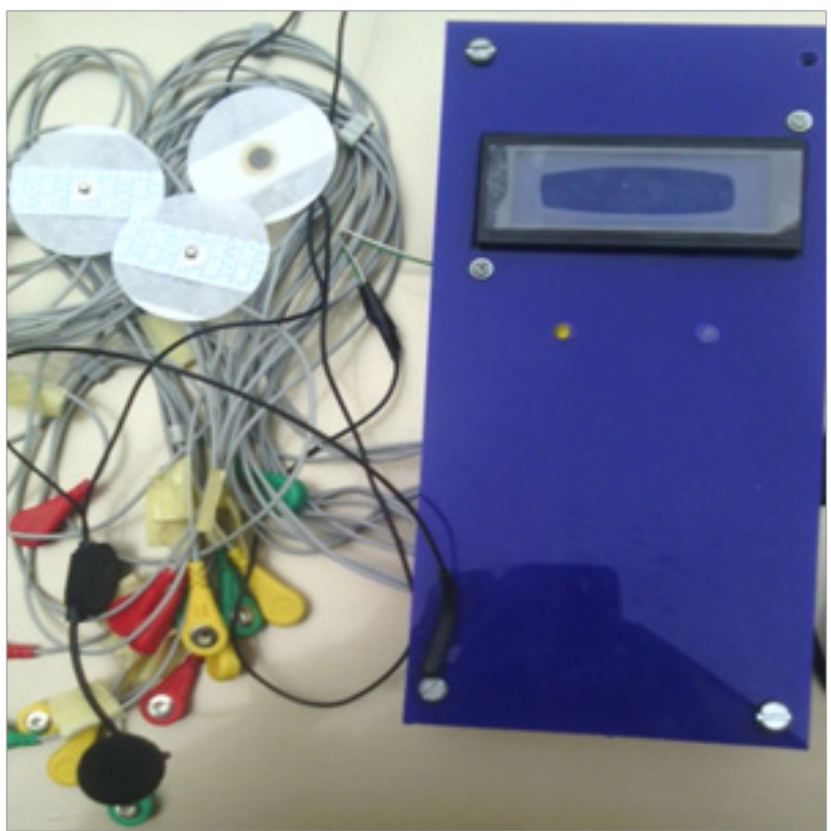

Figure 4 External view of the Transmitting Unit.

\section{Mode of operation}

The developed transmitting unit has three buttons apart from the power button. They are labelled F1, F2 and F3, with multitask assigned functions, all to be displayed on the LCD. F1 primary function is to set the system into configuration mode. This will enable the user to select any one of the four signals to be measured: The EMG, ECG and EEG signals are grouped into the first phase for compatibility while the second phase is allocated to speech signal. A second press on F1 will put the system in gain selection mode and another press on it will take the system to data acquisition mode. F2 on the other hand will take the system to scan mode at the initial press. Further singular press will put the system on a scan mode and another press will put the device on a data storage mode. F3 will display data fetching mode at the initial press. A second touch will affect the transmitting mode. When the device is set to scan by pressing the appropriate button, the electrode attached to the appropriate subject's body will convert the desired signals into voltage by the voltage divider network. The output is buffered by the operational amplifier circuit and fed into the microcontroller, where the signal is digitalized, pre-processed and stored in the well enhanced memory card. The stored data can at will be transmitted to the receiving unit, packets by packets, for acquiring, filtering and analysis.

\section{Disposable electrodes and the lead wires}

These electrodes are the latest innovation in biomedical measurement. Old forms are inserted into the skin and muscles through piercing. However the old method has to be discouraged in recent studies, because of infections and complications associated with incision and piercing. The type of the electrode employed in this research are H124SG pre-gelled silver chloride sensors because of their flexibility, reliability in EMG, ECG, EEG measurements and cost effectiveness. They are produced by placing a deposit of a thin layer working electrode material on a polymer film. Handling these electrodes should be a bit professional to ensure that the electrode is not detached. A disposable electrode when placed at the desired signal point can generate up to 3000 micro volts, to be transported through the lead wires. The lead wires are sensitive colored wires which the disposable electrodes, through clipping or clamping are attached, and they convey the signal inputs from the electrodes to the signal board for selection and amplification. Figure 5 shows the sample of electrodes and lead wires employed in this work.

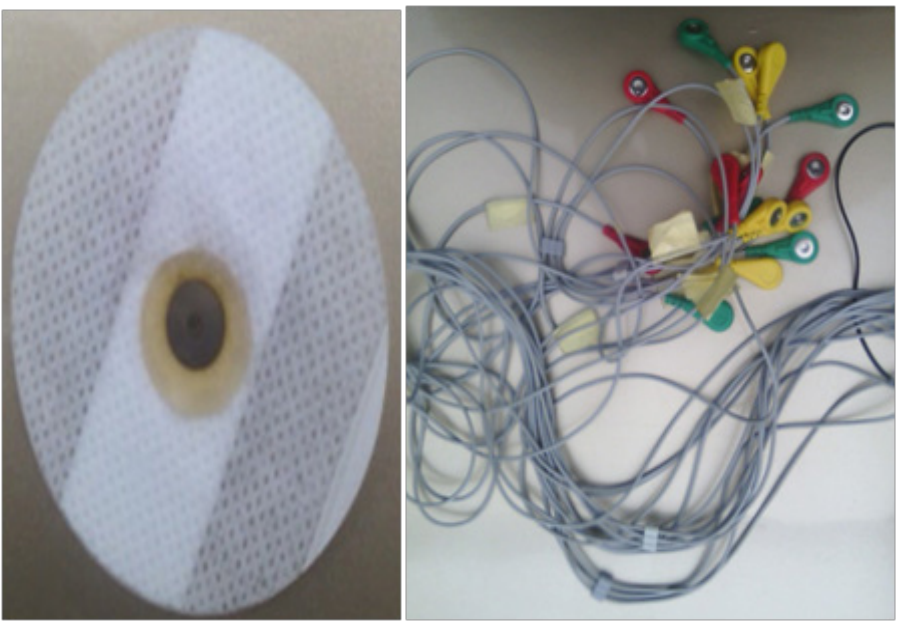

Figure 5 Sample of Electrode and LeadWires employed. 


\section{Signal pre-processing unit}

The signal pre-processing circuits is built from a voltage divider network (VDN) and 12 operational amplifiers. The VDN is made by connecting in series the lead sensors with $1000 \mathrm{Ohms}$ resistor as a potential divider. Each of the lead wires is inputted to an operational amplifier LC1024, where signals are amplified. LC1024 is a product of microchip technology Inc. It is memory accessed through simple SPI (serial Peripheral Interface) compatible. The output of each LC1024 is fed to the ADC inputs of PIC18F4450 microcontroller. LC1024 was selected because it has SPI compatible bus interface, $20 \mathrm{MHz}$ clock rate, write cycles and unlimited read with zero write time. It also has high reliability $128 \mathrm{~K}$ of 8 bit configuration and low power consumption technology of $3 \mathrm{~mA}$ at $5.5 \mathrm{~V}$ and 4 microampere standby current. The output signals of the opamps, connected as voltage follower circuit were buffered into the input of ADS1229 biosignal amplifier and LM3886 audio amplifier, the output of which was fed into PIC18F4450 microcontroller.

\section{Filtering techniques}

A butter worth low pass filter with a cut - off frequency $60 \mathrm{kHz}$, pass band gain 1.5 and capacitor of $1 \mu \mathrm{F}$ was designed as follows, for the biosignals which range from $10 \mathrm{~Hz}$ to $50 \mathrm{kHz}$.

$$
\begin{aligned}
& \text { From } \mathrm{f}_{\mathrm{c}}=\frac{1}{2 \pi R C} \\
& 60 \times 10^{3}=\frac{1}{2 \pi R \times 1 \times 10^{-6}}
\end{aligned}
$$$$
\mathrm{R}=26522.4 \Omega
$$

Since $26 \mathrm{k}$ is not available, then $\mathrm{R}$ is assumed to be $22 \mathrm{k} \Omega=\mathrm{R}_{\mathrm{l}}$

Amax $=1.5$ and assume $\mathrm{R}_{1}$ as $22 \mathrm{k} \Omega$

$A \max =1+R_{1} / R_{t}$

$$
\mathrm{R}_{\mathrm{t}}=11 \mathrm{k} \Omega
$$

\section{The PICI8F4450}

Microcontroller PIC18F4450 is one the family of PIC18XXXX from microchip technology with enhanced functions and cost effectiveness. It is a link between the hardware and the software. This low cost microcontroller was selected to overcome problems from using electronic components which consume higher power and prone to noise. The microcontroller has features such as high performance power managed mode, flexible oscillator structure up to $48 \mathrm{MHz}$, programmable Universal Serial bus with full speed of 12 megabytes per second, 10-Bit, Up to 13-Channel Analog-to-Digital Converter, up to $100 \mathrm{ks}$ per second sample rate and programmable time acquisition. The Pin and block diagram of PIC18F4450 are described by (Figure 3) (Figure 5) (Figure 6) and (Figure 3) (Figure 6) (Figure 7). Other special features that made the choice the microcontroller justifiable are:
i. C Compiler Optimized Architecture with Options
ii. Extended Instruction Set
iii. Flash Memory Retention: $>40$ Years
iv. Self-Programmable under Software Control

v. Priority Levels for Interrupts

vi. 8 x 8 Single-Cycle Hardware Multiplier

vii. Extended Watchdog Timer Programmable period from $4 \mathrm{~ms}$ to $131 \mathrm{~s}$ with

viii. Programmable Code Protection

ix. Single-Supply In-Circuit Serial Programming ${ }^{\text {TM }}$ (ICSPTM) via Two Pins

x. In-Circuit Debug (ICD) via Two Pins

xi. Optional Dedicated ICD/ICSP Port (44-pin TQFP devices only)

xii. Wide Operating Voltage Range (2.0V to $5.5 \mathrm{~V})$

The output of the programmed PIC is fed into the frequency modulation transmitter powered by 9 volts and employs frequency shift keying modulation scheme.

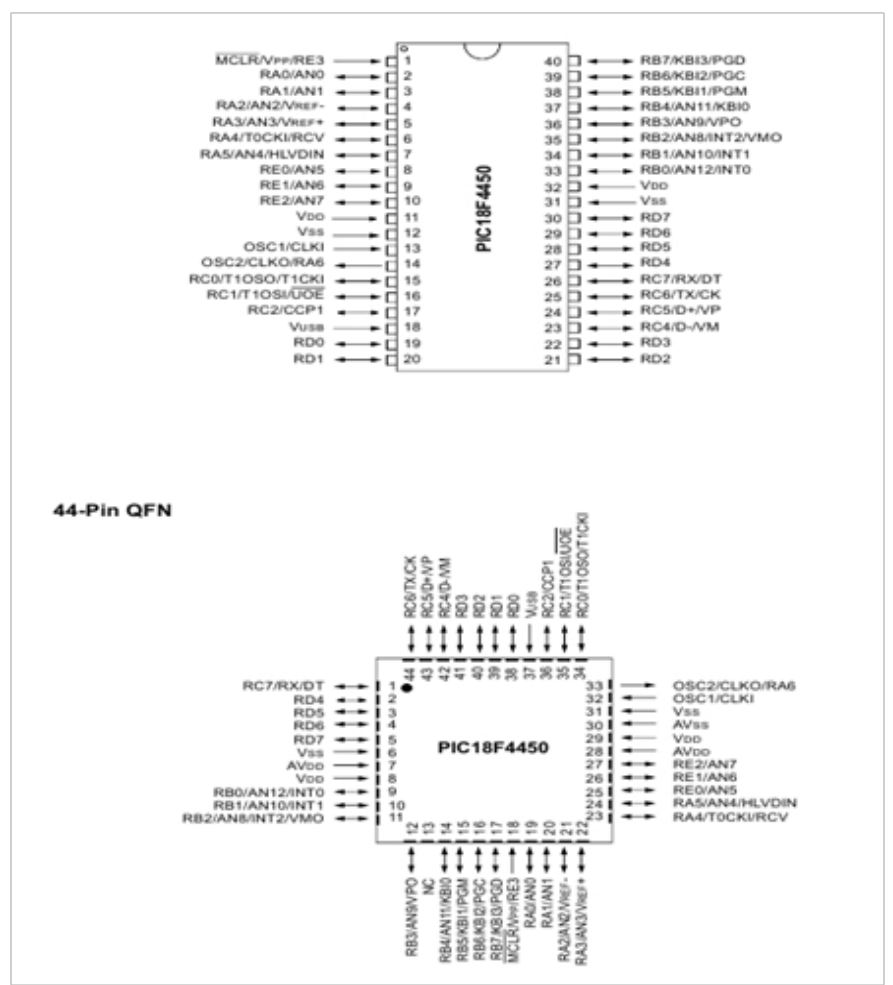

Figure 6 Pin Descriptions of PICI8F4450 Microcontroller (Data sheet at Microchip Technology 2012).

\section{The frequency modulation transmitter}

Current biosignal measurement in Nigerian hospitals is limited by inefficient transmission of biomedical information, right from the point of monitoring to the office of specialists assigned to interpret the data. Also some records of accidents have been recorded during the process of transporting (wheeling) patients from the admission wards to biosignal centers or laboratories where biomedical measurements are to be scanned. In some cases, patients placed on oxygen life support and drips have to be detached or disturbed while moving them to take tests and scans in the laboratories. In hospitals where there are internet facilities and consistent power supply, the radiologists depend mainly on sending scanned results through email to the medical 
personnel who requested the tests be made, or resolve to printing the results on paper. It is in the light of this that this research introduces the use of TX3A frequency modulation transmitter, with $869.85 \mathrm{MHz}$ carrier frequency for wireless communication and radiometry of all the measurable biomedical signals of focus. The transmitter is part of transmitter- receiver module of, data sheet 2009. The transmitterreceiver module is a miniaturized printed circuit board UHF radio pair. It enables the implementation of data link at distances up to 1000 meters in open environment, depending on other design components employed. The schematic and PCB for entire transmitting unit are shown in (Figure 8) (Figure 9). The circuit layout was developed using Diptrace Novaram 2011 software, for PCB building. Schematic components are laid on the interface of the software and tested in a soft mode. This was then printed and etched on a board, where the components are laid, traced, soldered and tested against partial contacts and dead joints.

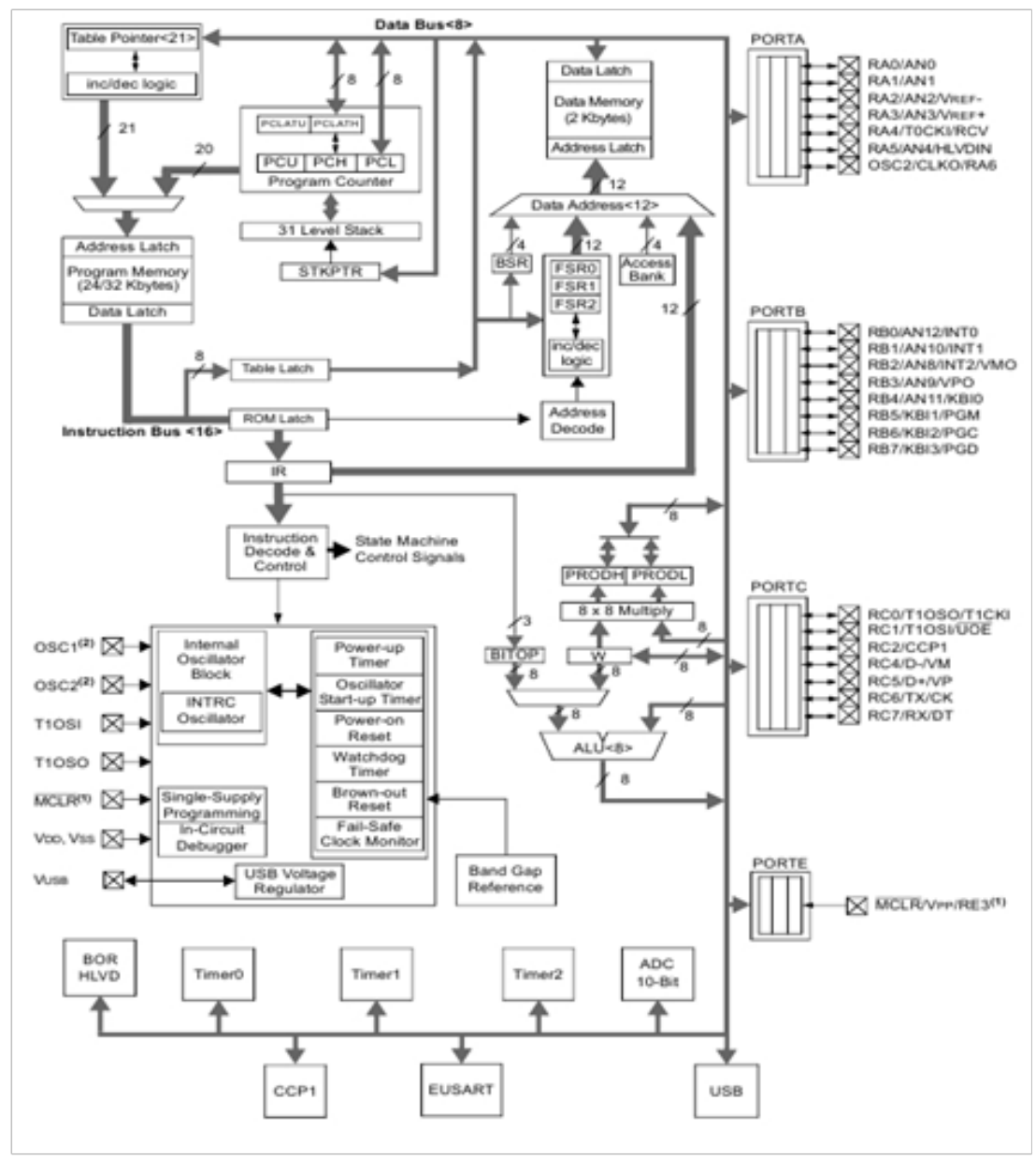

Figure 7 The Block Diagram of PICI8F4450.

\section{Audio amplifier unit}

A class A audio amplifier was designed with the following specifications;

$\mathrm{V}_{\mathrm{CEQ}}=9 \mathrm{~V}$ and $\mathrm{I}_{\mathrm{CQ}}=500 \mathrm{~mA}$ for the class-A, CE amplifier according to Figure 10, the output current varies by $\pm 300 \mathrm{~mA}$ and an input signal is applied at the base, the following parameters were calculated as follows;

(i) total dc power taken by the circuit as,

$\mathrm{P}_{\mathrm{in}(\mathrm{dc})}=\mathrm{V}_{\mathrm{CC}} \mathrm{I}_{\mathrm{CQ}}=9 \mathrm{~V}$ x $500 \mathrm{~mA}=4.5 \mathrm{~W}$

(ii) de power dissipated by the collector load

$\mathrm{P}_{\mathrm{Rc}(\mathrm{dc})}=\mathrm{I}_{\mathrm{CQ}}{ }^{2} \mathrm{R}_{\mathrm{C}}=(500 \mathrm{~mA})^{2} \times 470 \Omega=11.7 \mathrm{~W}$ (iii) ac power developed across the load

$$
\begin{aligned}
& \mathrm{P}_{\text {o(ac) }}=\mathrm{I}_{\mathrm{rms}}{ }^{2} \mathrm{R}_{\mathrm{C}} \\
& \mathrm{I}_{\mathrm{rms}}=\mathrm{I}_{\text {max }} / \sqrt{ } 2=300 \mathrm{~mA} / \sqrt{ } 2=212.13 \mathrm{~mA}=0.2121 \mathrm{~A} \\
& \mathrm{P}_{\text {o(ac) }}=0.2121^{2} \times 20=0.9 \mathrm{~W}
\end{aligned}
$$

(iv) power delivered to the transistor,

$$
\mathrm{P}_{\mathrm{tr}(\mathrm{dc})}=\mathrm{P}_{\mathrm{Rc}(\mathrm{dc})}-\mathrm{P}_{\mathrm{in}(\mathrm{dc})}=11.7 \mathrm{~W}-4.5 \mathrm{~W}=7.2 \mathrm{~W}
$$

(v) de power dissipated in transistor collector,

$\mathrm{P}_{\mathrm{c}(\mathrm{dc})}=\mathrm{P}_{\mathrm{tr}(\mathrm{dc})}-\mathrm{P}_{\mathrm{o}(\mathrm{ac})}=7.2 \mathrm{~W}-0.9 \mathrm{~W}=6.3 \mathrm{~W}$

Other essential parameters of Q1 like Voltage gain, Current gain, Input impedance, Output Impedance were also determined. 
R1 and R7 were used as pull up resistors for the switch and the value varied from 1 to $100 \mathrm{k}$ omhs

$\mathrm{R} 3$ and $\mathrm{R} 7$ were used for 5 volts reference for the ADC

$\mathrm{R} 8=47 \mathrm{ohm}$ and Q1 were used to switch the backlight at the LCD

Vout from the microcontroller pin $=5.0 \mathrm{~V}$

The maximum current that microcontroller pin can source $=10 \mathrm{~mA}$

So a voltage $<5.0 \mathrm{~V}, \mathrm{I}=10 \mathrm{~mA}$ is required to drive $\mathrm{Q} 1$

And going by Kirchhoff Law,

$\mathrm{I}=($ Vout $-\mathrm{VBE}) / \mathrm{R}$

And $\mathrm{VBE}=0.7$ Volt for silicon material
$\mathrm{R}=($ Vout $-\mathrm{VBE}) / \mathrm{I}$

$\mathrm{R}=(5.0-0.7) / 10 \mathrm{~mA}$

$\mathrm{R}=430 \mathrm{ohms}$

But there is no 430 ohms available, so $470 \mathrm{ohms}$ was chosen R6 helps in limiting the current flow through D9 to $3 \mathrm{~mA}$ D1 and D5 provided a drop of $1.8 \mathrm{~V}$ out of 5 volts.

$\mathrm{I}=$ Vout- 1.8 (VLED drops along the LED/R)

$=5-1.8 / 1 \mathrm{k}$

$3.2 / 1000=3.2 \mathrm{~mA}$

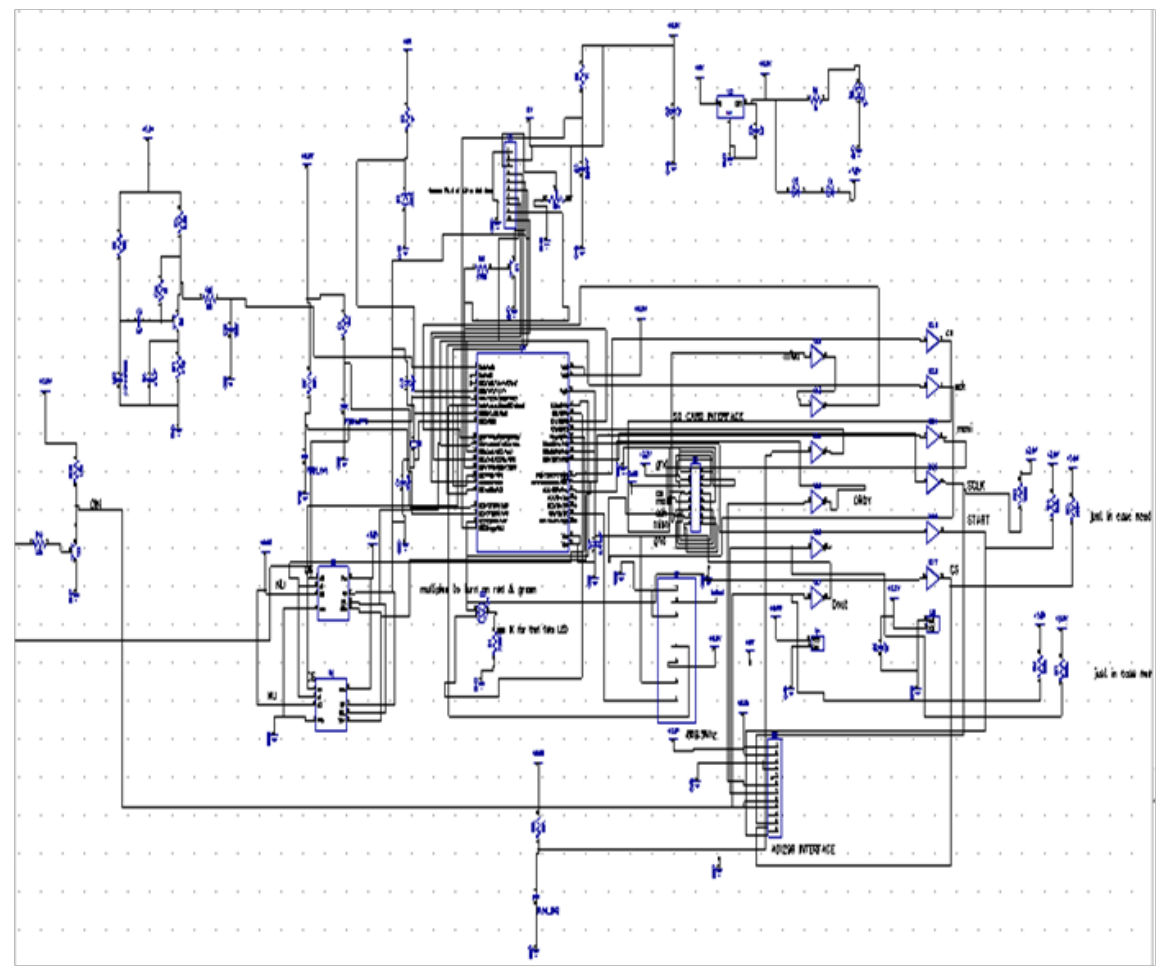

Figure 8 The Schematic Diagram of the Transmitting End imported from Dip-trace Location Layout.

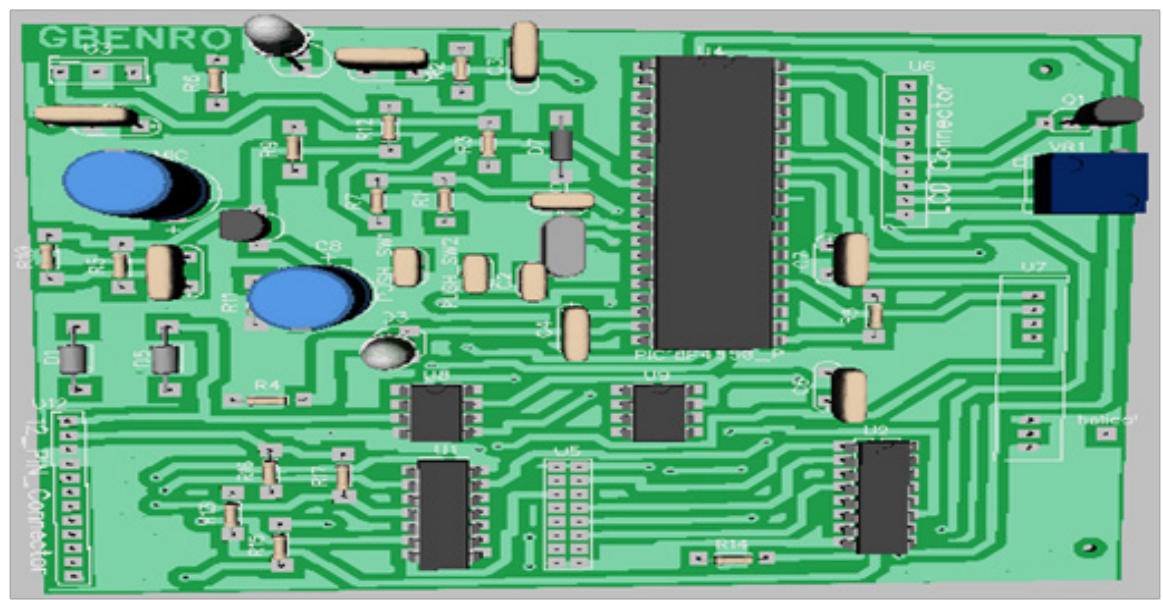

Figure 9 The PCB layout of the Transmitting End.

Citation: Ajani AS, Alade OM,Ajani OS, et al. Development of a mobile multimodal biosignal instrument for simultaneous measurement and analysis of four clinically relevant biosignals, obtained from both normal and pathological subjects. Int J Biosen Bioelectron. 2018;4(3):I48-I57. DOI: 10.15406/ijbsbe.2018.04.001 I7 


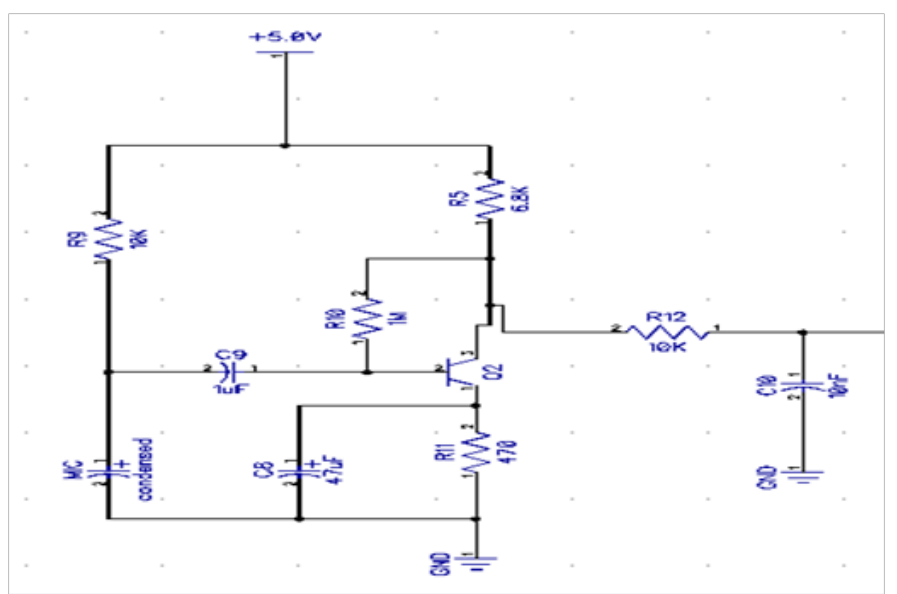

Figure I0 Audio Amplifier Unit.

\section{The receiving unit}

The receiving unit is stationary and it consists of RX3A FM receiver, RS232 driver, interfacing circuit, an improvised whip antenna. The block diagram of the receiving unit is shown in Figure 911A. RX3A is a double conversion super-heterodyne receiver which features a speedy action detection of carrier signal to indicate that signal is being received. The receiver has an inbuilt internal filtering circuit and slicing unit, which make it protected against random noise. The receiver output is the same as the transmitted coded width modulation signal. The circuit diagram of the stationary receiving unit is shown in Figure 11B. Different types of antennas are prescribed for use with the transmission and receiving network, however, whip antenna was improvised because of size, good performance, immunity to effects of proximity and high coverage. It was made from a coated $3 \mathrm{~mm}$ diameter copper wire, connected to the second pin and first pin of the transmitter and the receiver respectively. The receiving end of the network is shown in Figure 12.

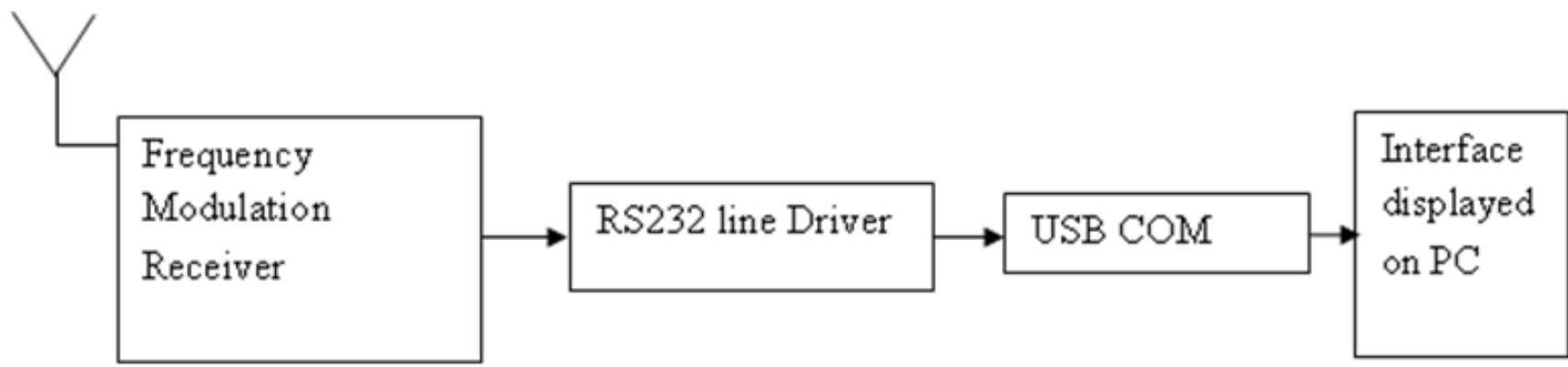

I (A) Block Diagram of the Receiving End.

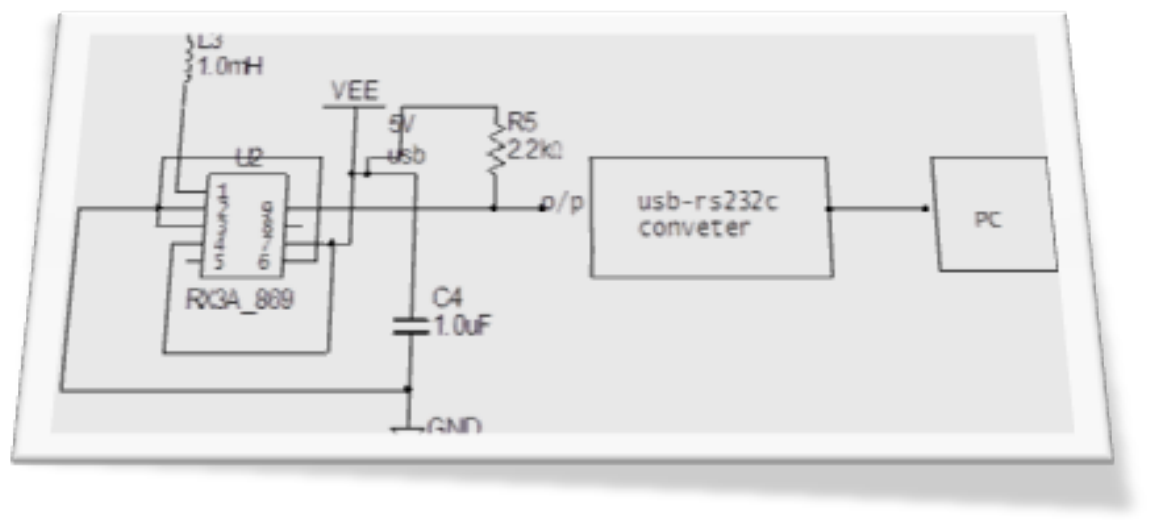

I I (B) Receiving Circuit Network.

Figure II Block and Circuit Diagram of the Receiving unit.

\section{Results and discussion}

\section{Setup of the biosignal multimodal instrumentation}

Figure 13 shows the complete biosignal system units. The whole setup consists of the mobile transmitting unit already fixed with the lead wire carrying the disposable electrode; the mobile receiving unit connected to the computer through the USB, a 64 bit GO RAM Personal Computer for output analysis and the distance tape for coverage determination.

\section{Measurement of biosignal parameters}

The method used in this research to measure the biosignal data and obtain the signal information involves obtaining official permission from five (5) different university teaching hospitals located at the South-western part of Nigeria, for access to use some of the patients admitted in their medical wards. The target subjects are patients with neuromuscular disorder, stroke, Parkinson disease, Cardiac arrest and other related pathological cases. Carrying along the health and medical personnel in charge of each ward, the patients to be sampled 
were approached and enlightened on the merit and importance of the research, appealing to their cooperation and support. In some cases the machine will be requested by the patients, to be tested on one of the research crews, to avoid phobia and ascertain there were no side effects. There were cases where the radiologists were requested to assist in mounting the electrodes at the appropriate target signal location in the patients' body. One of the limitations encountered at this stage is the inability to use the device to monitor the ECG information of female patients because it involves complete removal of dress whereas the researcher is a male. A female nurse was trained to use the machine in a private ward for female patients, to address this limitation. Patients with stroke are the perfect specimens for the examination of the four investigated biosignals. The transmitter side is connected to the patient by positioning the electrodes at the target points. The surfaces of the points of target were cleaned using alcohol, water and cotton wool. The signals were then captured by operating the transmitting end. The data is then stored in the embedded SD card of the transmitter, for immediate data transfer and upward transmission. The speech signal was captured by telling the patient to pronounce or read some sentences written on a sheet of paper. EMG signal was capture by requesting the patient to flex the muscles in the area of target for the EMG. The stored data is immediately sent to the receiver, already connected to the $\mathrm{PC}$ for data acquiring and Analysis. Figure 14-17 shows some of the samples taken during data capturing.
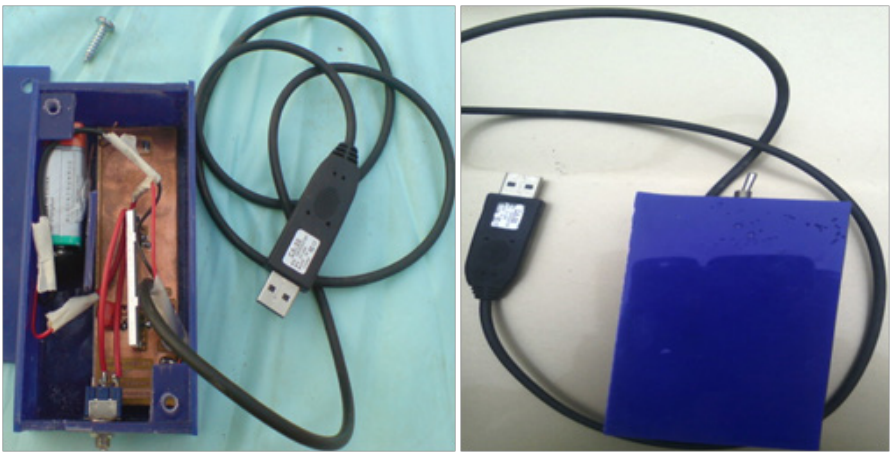

Figure 12 The diagram of the complete receiving end.

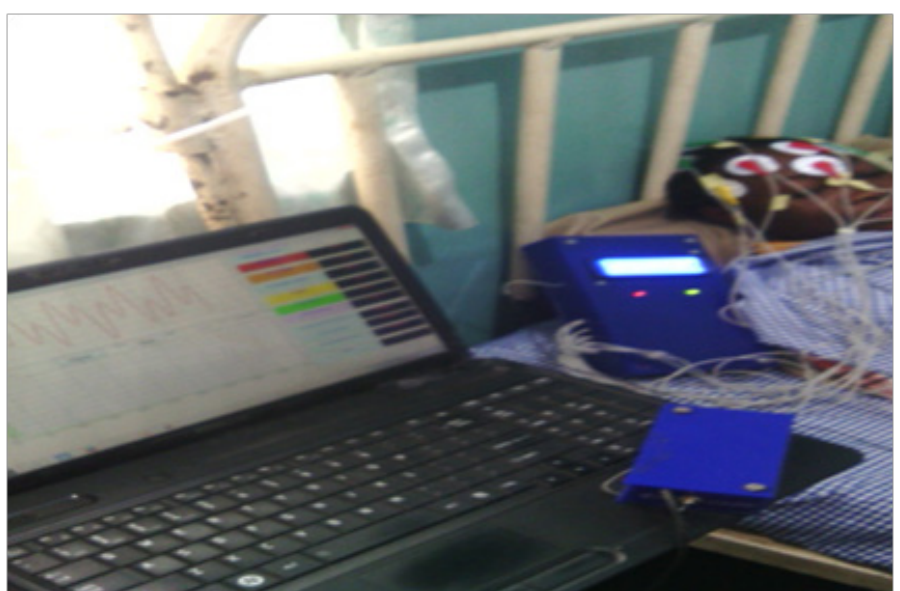

Figure I 3 Experimental setup for Biosignal Capturing.

\section{Results acquired through biosignal instrument}

Real-time data acquisition directly from the source was carried out by direct electrical connection to instruments. This overcomes the need for experts to monitor, encode and enter data manually. Sensors attached to patient's body permits biomedical signals to be converted into electrical signals which are then sent into a computer. The signals were periodically picked and converted to digital form for storage and analysis. Data acquisition and analysis automation are very important in monitoring patients, because naturally occuring signals are in analogue form, hence the need for analogue to digital converter. ${ }^{2}$ How closely the digital data matches the original analogue signals is determined by two important factors; the precision with which the signals are recorded and the frequency at which signals are sampled. As a general rule, at least twice as frequent as the highest frequency component needed from a signal must be sampled. Just as in ECG measurement, the basic repetitive frequency is at few samples per second, but that of the QRS complex contains useful frequency components in the order of $150 \mathrm{~Hz} .{ }^{3}$ Hence, data sampling rate should be at least three hundred measurements per second (300 Sps). $500 \mathrm{Sps}$ was chosen as minimum in this research and this was done in packtes of signals. Transmitted signals are acquired by selecting the required signal and pressing the Acquire command on the biosignal interface.

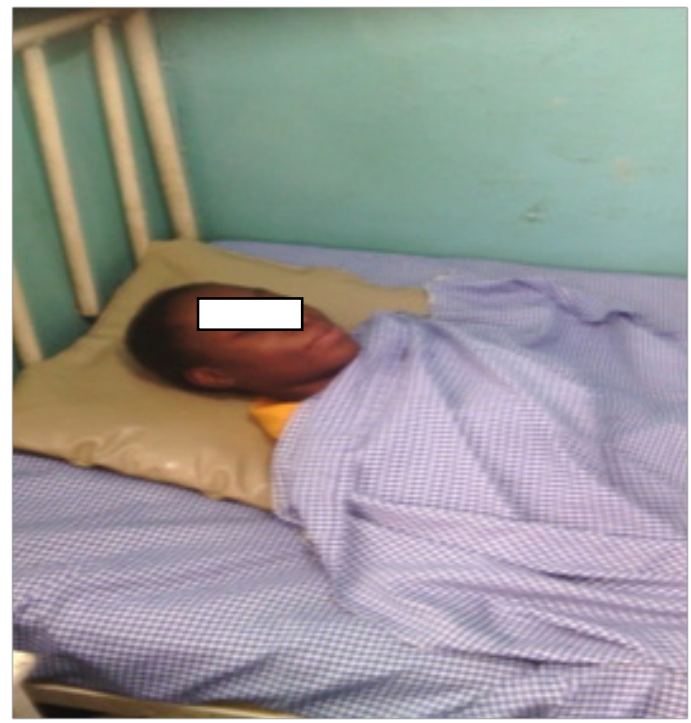

Figure 14 Preparing the Subject for Data Capturing.
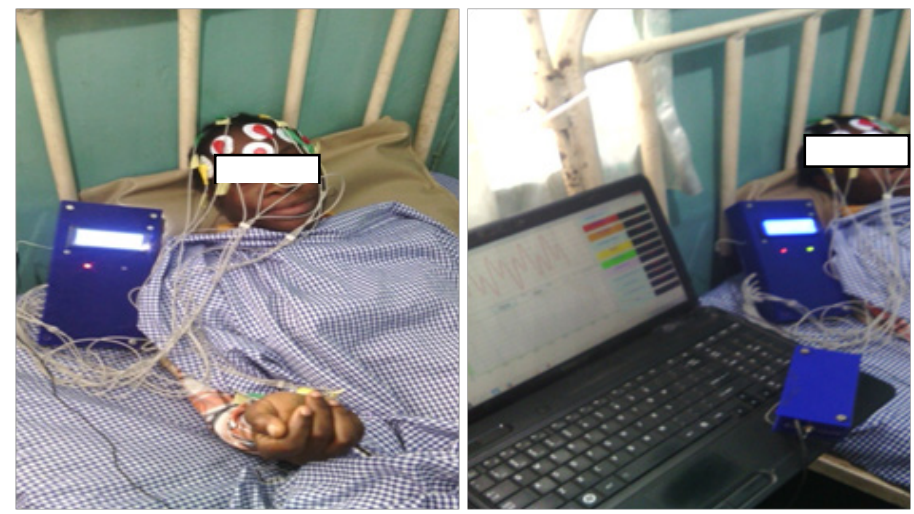

Figure 15 Data Capturing Mode for EEG. 

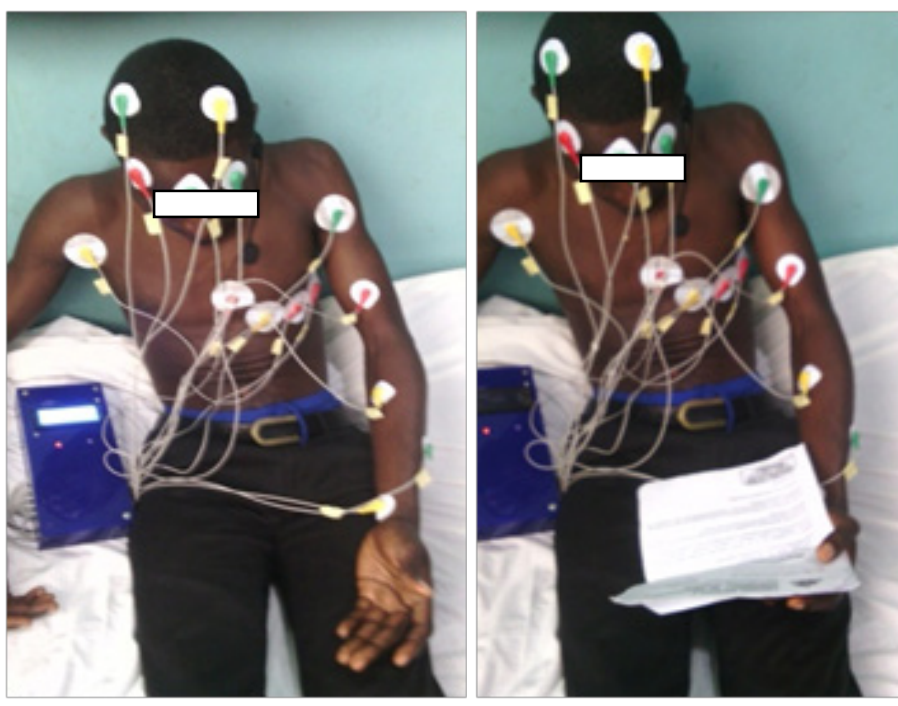

Figure I6 Data Capturing Mode for EEG, ECG, EMG and Speech.

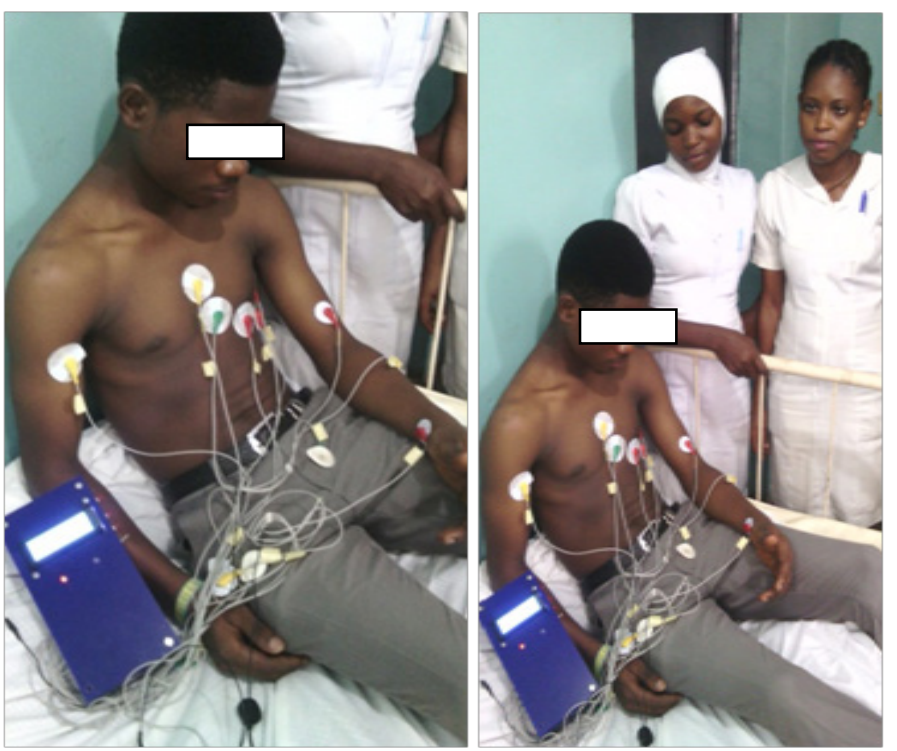

Figure 17 Data Capturing Mode for ECG and EMG.

\section{Conclusion}

Computer-based multimodal digital biosignal instrumentation for acquisition and analysis of EMG, ECG, EEG and Speech has been developed in this research. The whole instrument is made up of three sections: the $9 \mathrm{~V}$ battery powered mobile transmitting unit, the receiving unit and the user friendly interfaces. The mobile transmitting unit consists of improvised disposable electrode-lead wire transduction network, which converts biosignals from human body to electrical signals. Processing circuit was performed here by signal conditioning circuit for linear operation of amplification on the transducer output, before transmission takes place. Signals were picked by appropriately attaching the sensors to the target locations on subject's body. The transmission link between the sensor side and the stationary processing unit overcomes the problems associated with most clinical scanning devices. The link provides the opportunity to continuously monitor patients on admission without wheeling them around to laboratories. It provides electrical isolation by using low voltage battery thus preventing electrical shocks and power failure. The receiving end received data at the transmitted frequency of $869.85 \mathrm{MHz}$ with minimized distortion and the data can be acquired and analysed with aid of the user designed biosignal interface, having the data capturing mode and analysis mode. The data can be analysed in either time domain or frequency domain analysis with fast transfer mode and the test analysis taken showed the device can be used as a clinical and research tool.

\section{Recommendations}

One of the limitations encountered in this study is the instability of the sensors due to dryness of the surface gel. Thus further work on the sensor performance and size reduction is highly recommended by the researcher. Subsequent works in the area of this research should also take into cognizance the miniaturization of the transmitting component of the system to minimize imposition on the patients. The researcher suggests a lead wire-free sensor network for the transmission of data; as it will be worthwhile if the lead wire link can be scrapped by designing sensors capable of being pre-processed to send picked values directly to the transmitter. The developments and designs of medical electronic instrumentations are advised to be encouraged and priotised by the government of Nigeria and other policy makers, to ensure our indigenous technology better develop and reduce unwarranted spending in foreign hospitals. The developed biosignal system can find applications in areas such as diagnosis of ischemia, arrhythmia, conduction defects, sleep studies, seizure detection, cortical mapping, muscle functions, neuromuscular diseases, prosthesis and in other medical investigations.

\section{Acknowledgements}

None.

\section{Conflict of interest}

Author declares that there is no conflict of interest.

\section{References}

1. WHO. World Health Organization, on Cardiovascular Disease, global cause of death. 2018.

2. Gardner RM, Shabot MM. Patient-Monitoring Systems, Biomedical Informatics. 3rd ed. New York: Springer; 2006.

3. Trägårdh E, Claesson M, Wagner GS, et al. Reduced highfrequency QRS components in electrocardiogram leads facing an area of the heart with intraventricular conduction delay due to bundle branch block. Journal of Electrocardiology. 2007;40(2):127-132.

4. Agrafioti F, Hatzinakos D. ECG biometric analysis in cardiac irregularity conditions. Signal, Image and Video Processing. 2008:1863-1703.

5. Ampornaramveth V, Hasanuzzaman M, Zhang T, et al. Gesture based human-robot interaction using a frame based software platform. IEEE Int Conf Man Cybernet. 2004;4:2883-2888.

6. Alade MO, Ajani AS. Performance evaluation of UHF wireless digital gait monitoring instrumentation for long range data transmission. International Journal of Advanced Research in Electrical, Electronics and Instrumentation Engineering. 2013;2(1):696-704.

7. Atkielski A. Schematic diagram of normal sinus rhythm for a human heart as seen on ECG. 2010.

8. Barkhaus P. EMG Evaluation of the Motor Unit Electrophysiologic Biopsy. 2012. 
9. Blascovich J, Seery M, Mugridge C, et al. Predicting athletic performance from cardiovascular indexes of challenge and threat. Journal of Experimental Social Psychology. 2004;4(5):683-688.

10. Camm AJ, Macfarlane PW, Van O Ostrom A, et al. Heart rate variability: Standards of measurement, physiological interpretation, and clinical European Heart Journal. 1996;17:354-381.

11. Chan FHY, Yang YS, Lam FK, et al. Fuzzy EMG classification for prosthesis control. IEEE Transactions Rehabilitation Engineering. 2000;8(3):305-311.

12. Cram JR, Kasman GS, Holtz J. Introduction to Surface Electromyography. Gaithersburg, Maryland: Aspen Publishers Inc; 1998.

13. De Luca CJ. Electromyography, in Encyclopedia of Medical Devices and Instrumentation. In: Webster JG, Editor. New York: Wiley; 1996. p. 195-226.

14. Draper H, Peffer C, Stallmann C, et al. The corrected orthogonal electrocardiogram and vectorcardiogram in 510 normal men (Frank leadsystem). Circulation. 1964;30:853-864.

15. Fang J, Agarwal GC, Shahani BT. Decomposition of EMG signals by wavelet spectrum matching. Procedures of the 19th Annual International Conference of the IEEE Engineering in Medicine and Biology Society; Chicago, IL, USA; 1997. p. 1253-1256.

16. Gonzalez RC, Woods RE, Eddins SL. Digital image processing using MATLAB' (Pearson Prentice-Hall). 2004.

17. Graffiti A, Hatz L. ECG based recognition using second order statistics. In Proceedings of 6th Annual Communication Networks and Services Research Conference; UK: Halifax; 2008. p. 82-87.

18. Hodgins D. Human Motion Analysis. Medical Device Technology. Codicote, UK: European Technology for Business Ltd; 2008.

19. Marius D, Pennathur S, Rose K. Face detection using colour thresholding, and Eigen image template matching', EE368: Digital Image Processing Project. 2003.

20. Merlo A, Farina D. A Fast and Reliable Technique for Muscle Activity Detection from Surface EMG Signals. IEEE Trans Biomed Eng 2003;50(3):316-323.
21. Micera S, Vannozzi G, Sabatini AM, et al. Improving detection of muscle activation intervals. IEEE Engineering in Medicine and Biology Magazine. 20001;20(6):38-46.

22. Muhammad Ibn Ibrahimy. Biomedical Signal Processing and Application. Proceeding of the 2010 international Conference on Industrial Engineering and Operations Management. Dhaka, Bangladesh; 2010 .

23. Pilkington T, Barr R, Rogers CL. Effect of conductivity interfaces in electrocardio- graphy. New York: Springer; 2006. p. 637-643.

24. Richter M1, Friedrich A, Gendolla GH. Task difficulty effects on cardiac activity. Psychophysiology. 2008;45:869-875.

25. Roger GM. Clinical Electrocardiography and Arrhythmias. USA: 2004. p. 34-41.

26. Shan C, Wei Y, Qiu X, et al. Gesture recognition using temporal template based trajectories'. Proc 17th Int Conf Pattern Recognition. 2004;3:954 957

27. Shirai C, Takai T, Nariai M. A time-frequency approach to evaluate electromyographic recordings. Proceedings of Fifth Annual IEEE Symposium on Computer-Based Medical Systems. 2004;520-527.

28. Song J, Michael RL. A Hough transform based line recognition method utilizing both parameter space and image space. Pattern Recognition. 2005;38(4):539-552.

29. Van Bemmel J, Musen M. Handbook of medical informatics. 2nd ed. Houten Digem: Springer; 1997. p. 336-345.

30. Webster JG. Medical Instrumentation: Application and Design. 3rd ed. New York: Wiley; 1998

31. Yang J, Min Zhang, Shu-bang Li, et al. A New Filtering Algorithm for Removing Salt-andPepper Noise. InternationalConference on Environmental Science and Information Application Technology. 2009; 1:355-358.

32. Zennaro D, Welling P, Koch VM, et al. A Software Package for the Decomposition of Long-Term Multichannel EMG Signal Using Wavelet Coefficients. IEEE Trans Biomed Eng. 2003;50(1):58-69. 